\title{
ANÁLISIS DE TÉCNICAS QUE PERMITAN INCREMENTAR LA ESTABILIDAD DE CARROTANQUES
}

\section{ANALYSIS OF TECHNIQUES THAT ALLOW TO INCREASE THE STABILITY OF ROAD TANKERS}

\author{
PhD. Gonzalo G Moreno C*, PhD. Juan Carlos Serrano* \\ MSc. Edwin Espinel Blanco** \\ * Universidad de Pamplona, Facultad de Ingenierías y Arquitectura, \\ Programa de Ingeniería Mecánica, \\ Grupo de Investigación en Ingeniería Mecánica de la Universidad de Pamplona (GIMUP). \\ Pamplona-Colombia, Km 1 vía Bucaramanga. \\ Tel.: (+57 7) 568 5303, Fax: (+57 7) 568 5303, Ext. 144 \\ E-mail: gmoren, jcserrano@unipamplona.edu.co \\ ** Universidad Francisco de Paula Santander - Ocaña, \\ Programa de Ingeniería Mecánica, \\ Grupo de Investigación en Tecnología y Desarrollo en Ingeniería - GITYD \\ Ocaña - Colombia \\ Tel.: (+57 7) 569 0088, Ext. 210 \\ eeespinelb@ufpso.edu.co
}

\begin{abstract}
Resumen: Los vehículos pesados debido a su gran dimensión tienden a tener problemas de estabilidad cuando se encuentran transitando por curvas, esto se debe en gran parte a que el centro de gravedad de la carga transportada en muy alto, adicionalmente, si se tiene en cuenta la rigidez de los neumáticos y de la suspensión la estabilidad se ve mayormente afectada. Otro factor que afecta seriamente la estabilidad son las cargas liquidas, las cuales debido a su movimiento natural cuando están bajo la acción de una aceleración lateral (fuerza centrípeta) afectan aún más la estabilidad de estos vehículos. Teniendo en cuenta estas especificaciones, en el presente artículo y mediante un estudio de caso, se detallan algunas técnicas que permitirían mejorar la estabilidad de este tipo de vehículos.
\end{abstract}

Palabras clave: Carro-tanque, estabilidad, nivel de llenado.

\begin{abstract}
Heavy vehicles due to their large size tend to have stability problems when they are traveling through curves, this is due that the center of gravity of the load being transported very high, additionally, if the stiffness of the tires and the suspension are taken into account the stability is mostly affected. Another factor that seriously affects stability is liquid loads, which due to their natural movement when they are under the action of a lateral acceleration (centripetal force) further affect the stability of these vehicles. Taking these specifications into account, in this article and through a case study, some techniques are detailed that would allow to improve the stability of this type of vehicles.
\end{abstract}

Keywords: Road tanker, stability, filling level. 


\section{INTRODUCCIÓN}

El transporte terrestre de cargas liquidas representa hoy en día un gran reto ingenieril, ya que su movimiento supone cambios drásticos y repentinos de la distribución de carga en el vehículo. Esta característica especial, es tenida en cuenta en estudios de estabilidad vehicular, en los cuales se analiza como el nivel de llenado del carro-tanque afecta la estabilidad, según Ranganathan, (1993); Winkler, (2000) y Prem et al, (2006) para niveles de llenado entre el $40 \%$ y $60 \%$ el movimiento del líquido transportado hace que el vehículo sea más propenso al volcamiento, lo cual hace que el conductor debe tener especial cuidado al realizar determinadas maniobras; (J Plaza, M Núñez, 2017); (R García - León, et al., 2016).

En este sentido, y tomando como base él estudió de estabilidad desarrollado por Moreno et al, (2017a, 2017b) en el presente artículo se hace una comparación mediante un estudio de caso, de cómo ciertos cambios estructurales pueden mejorar la estabilidad del vehículo; (L Tangarife et al., 2017).

Por consiguiente, el en presente artículo se hace un análisis bidimensional del movimiento de la carga (fluido) en un tanque de sección transversal circular bajo la acción de una aceleración lateral (Sección 2), seguidamente mediante un análisis de estabilidad se desarrolla el factor de estabilidad bidimensional (Static Rollover Threshold - SRT) para este tipo de vehículos en la Sección 3, en la Sección 4 se analiza mediante un estudio de caso como algunas suposiciones estructurales pueden propiciar un aumento en la estabilidad del vehículo, lo cual sirve como base para orientar trabajos futuros en el área, por último, las conclusiones de este trabajo son dadas en la Sección 5.

\section{MOVIMIENTO DEL FLUIDO CON ACELERACIÓN UNIFORME}

Las ecuaciones que describen el movimiento lineal de un fluido incompresible dentro de un tanque circular sometido a aceleración uniforme como el mostrado en la ;Error! No se encuentra el origen de la referencia., son las ecuaciones de continuidad y la ecuación de conservación de momentum (Çengel \& Cimbala, 2006), lo cual es presentado en las Ec. 1 y 2 .

$$
\nabla \cdot v=0
$$

y

$$
\rho\left(\frac{\partial v}{\partial t}+v \cdot \nabla v\right)=-\nabla P+\rho g+\mu \nabla^{2} v
$$

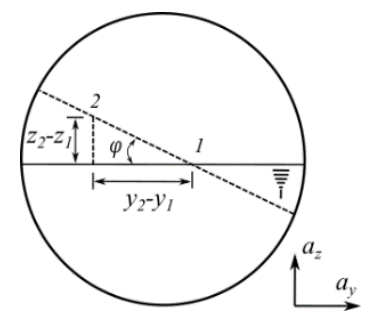

Figura 1. Fluido en aceleración en tanque de sección transversal circular

Donde $v$ es la velocidad del fluido $[\mathrm{m} / \mathrm{s}$ ], $\mathrm{P}$ es la presión del fluido $[\mathrm{Pa}], \rho$ es la densidad del fluido $\left[\mathrm{kg} / \mathrm{m}^{3}\right], \mu$ es la viscosidad del fluido $[\mathrm{Pa} \cdot \mathrm{s}]$ y $\mathrm{g}$ es la aceleración debida a la gravedad $\left[\mathrm{m} / \mathrm{s}^{2}\right]$. Cuando el fluido es sometido a una aceleración lateral, cada elemento del fluido en el tanque experimenta la misma aceleración, además su velocidad $v$ es función del tiempo más no de la posición, la Ec. (2) puede ser reescrita como:

$$
\rho \frac{D v}{D t}=-\nabla P+\rho g+\mu \nabla^{2} v
$$

Donde $D / D t$ es la derivada substancial. Para un flujo uniformemente acelerado $D / D t=a \mathrm{y} \nabla^{2} v=0$, lo cual reduce la Ec.(3) a

$$
\rho a=-\nabla P+\rho g
$$

La Ec. (4) puede ser expresada explícitamente como:

$$
\begin{aligned}
& \frac{\partial P}{\partial x} \vec{i}+\frac{\partial P}{\partial y} \vec{j}+\frac{\partial P}{\partial z} \vec{k}+\rho g \vec{k}= \\
& -\rho\left(a_{x} \vec{i}+a_{y} \vec{j}+a_{z} \vec{k}\right)
\end{aligned}
$$

Considerando el movimiento del fluido en el plano $y-z$, la Ec. (5) se reduce a:

$$
d P=-\rho a_{y} d y-\rho\left(g+a_{z}\right) d z
$$

Cuando un tanque es uniformemente acelerado en la dirección $y$, inicialmente la superficie libre se moverá hacia atrás y adelante, después de algún tiempo este movimiento desaparece, pasando de este estado transitorio a un movimiento uniformemente acelerado. La posición de la 
superficie del líquido en un tanque uniformemente acelerado es mostrada en la Fig.1. Para $\rho=c t e$, la diferencia de presión entre dos puntos 1 y 2 en el fluido es determinada por integración, con lo que la Ec.(6) se transforma en:

$$
P_{2}-P_{1}=-\rho a_{y}\left(y_{2}-y_{1}\right)-\rho\left(g+a_{z}\right)\left(z_{2}-z_{1}\right)
$$

El aumento vertical de la superficie libre en el punto 2 relativo al punto 1 puede ser determinado seleccionando estos puntos sobre la superficie libre donde $P_{2}=P_{1}$ y solucionando la Ec.(7)

$$
\nabla z=z_{2}-z_{1}=-\frac{a_{y}}{g+a_{z}}\left(y_{2}-y_{1}\right)
$$

En un fluido incompresible con aceleración uniforme en movimiento lineal, las líneas de presión constante son paralelas a la superficie cuya pendiente en el plano $y-z$ con $a_{z}=0$ puede ser determinada mediante:

$$
\text { pendiente }=-\frac{a_{y}}{g+a_{z}}=-\frac{a_{y}}{g}=\tan \varphi
$$

Con el objetivo de determinar el centro de gravedad del fluido $\left(\mathrm{CG}_{2}\right)$ de un tanque de sección transversal circular, sometido a aceleración uniforme y con determinado porcentaje de llenado, las coordenadas del centro de gravedad se determinan mediante la solución de:

$$
\begin{aligned}
& \bar{x}=\frac{1}{A} \iint_{A} x d A \\
& \bar{y}=\frac{1}{A} \iint_{A} x d A
\end{aligned}
$$

\section{ANÁLISIS DE ESTABILIDAD DEL VEHÍCULO}

Para este análisis se utilizó la representación bidimensional de un carro tanque de sección circular, el cual cuenta con dos centros de gravedad, en el primero $\left(C G_{1}\right)$ se ubica la carga fija o no móvil del vehículo y en el segundo $\left(C G_{2}\right)$ se ubica la carga del fluido transportado, adicionalmente, cada una de estas cargas tiene una carga centrípeta asociada $\left(\begin{array}{lll}m_{1} a_{y} & y & m_{2} a_{y}\end{array}\right)$, como se observa en la Figura 2. Adicionalmente, del análisis cuasi-estático del modelo, el cual utiliza como punto de referencia para la toma de momentos el punto de contacto de la rueda exterior a la curva con el suelo (punto A) (Figura 2) se obtiene la Ecuación (11).

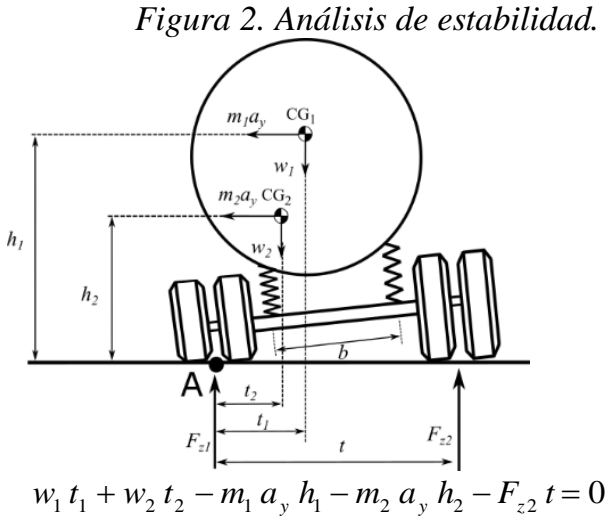

De la Ec. 11 y de la Figura 7 se tiene que: $t_{1}$ es la distancia lateral desde el punto de contacto de la rueda externa a la curva hasta en $C G_{1}, t_{2}$ es la distancia lateral desde el punto de apoyo de la rueda externa a la curva hasta el $C G_{2}, h_{1}$ es la altura del $C G_{l}, h_{2}$ es la altura del $C G_{2}, t$ es la distancia lateral entre las ruedas o ancho de vía, b es la distancia lateral de separación de la suspensión, y $F_{z i}$ son las fuerzas normales de apoyo del vehículo. Teniendo en cuenta que, en el umbral de vuelco dinámico del vehículo, la fuerza normal de apoyo de la rueda interna a la curva tienda a cero $\left(F_{z 2}=0\right)$, reorganizando la ecuación se obtiene que el factor de estabilidad bidimensional ( $S R T$ ) para un vehículo tipo carro-tanque, el cual se expresa mediante la siguiente ecuación:

$$
S R T=\frac{a_{y}}{g}=\frac{m_{1} t_{1}+m_{2} t_{2}}{m_{1} h_{1}-m_{2} h_{2}}
$$

Esta ecuación nos indica que el factor de estabilidad no depende únicamente de la posición de los centros de gravedad, sino que también es función de los pesos que intervienen en el vehículo.

\section{CASO DE ESTUDIO}

Para este estudio se utilizó el modelo de un vehículo pesado tipo carro-tanque (Figura 2) y con las especificaciones de la Tabla 1. Para el cálculo del factor de estabilidad se tuvo en cuenta la norma ISO14792 - Steady State Circular Test (2011) y que por seguridad vial la transferencia lateral de carga no debe ser mayor del 60\% (Woodrooffe et al, 2016; Walker \& Person, 1987), una vez implementado el modelo, se aumentaron gradualmente las fuerzas centrípetas $\left(m_{1} a_{y}\right.$ y $\left.m_{2} a_{y}\right)$ hasta que la transferencia de carga lateral fuera la recomendada. 
Tabla 1: Parámetros - vehículo pesado

\begin{tabular}{ccc}
\hline Parámetro & Cantidad & Unidades \\
\hline $\begin{array}{c}\text { Peso vacío }\left(\boldsymbol{w}_{1}\right) \\
\text { Peso del líquido } \\
\text { transportado } \\
\left(\boldsymbol{w}_{2}\right)(\text { agua) }\end{array}$ & 60 & $\mathrm{kN}$ \\
$\begin{array}{c}\text { Rigidez combinada de } \\
\text { la suspensión }(3 \text { ejes) } \\
\text { Rigidez combinada de } \\
\text { los neumáticos }\end{array}$ & 5400 & $\mathrm{kN}$ \\
$\begin{array}{c}\text { Distancia lateral entre } \\
\text { las ruedas }(\boldsymbol{t})\end{array}$ & 1.86 & $\mathrm{kN} / \mathrm{m}$ \\
$\begin{array}{c}\text { Diámetro del tanque } \\
\text { Altura inicial del } \boldsymbol{C} \boldsymbol{G}_{\boldsymbol{1}} \\
\quad\left(\boldsymbol{h}_{\boldsymbol{1}}\right)\end{array}$ & 1.015 & $\mathrm{~m}$ \\
$\begin{array}{c}\text { Altura inicial del } \\
\text { centro geométrico del } \\
\text { tanque }\end{array}$ & 2.85 & $\mathrm{~m}$ \\
$\begin{array}{c}\text { Distancia lateral entre } \\
\text { los muelles de la } \\
\text { suspensión }(\boldsymbol{b})\end{array}$ & 0.95 & $\mathrm{~m}$ \\
\hline
\end{tabular}

Inicialmente se hacen mediciones de la estabilidad del vehículo para niveles de llenado del $40 \%$ y del $60 \%$, posteriormente estas mediciones son comparadas con mediciones similares hechas para el mismo vehículo en el cual se supone que no hay movimiento del fluido. Esta comparación permite observar (Figuras 3 y 4 ) que la estabilidad del vehículo se ve incrementada en alrededor 0.01356. Este aumento en el factor de estabilidad supone una mayor seguridad en el tránsito de estos vehículos al tomar una curva.

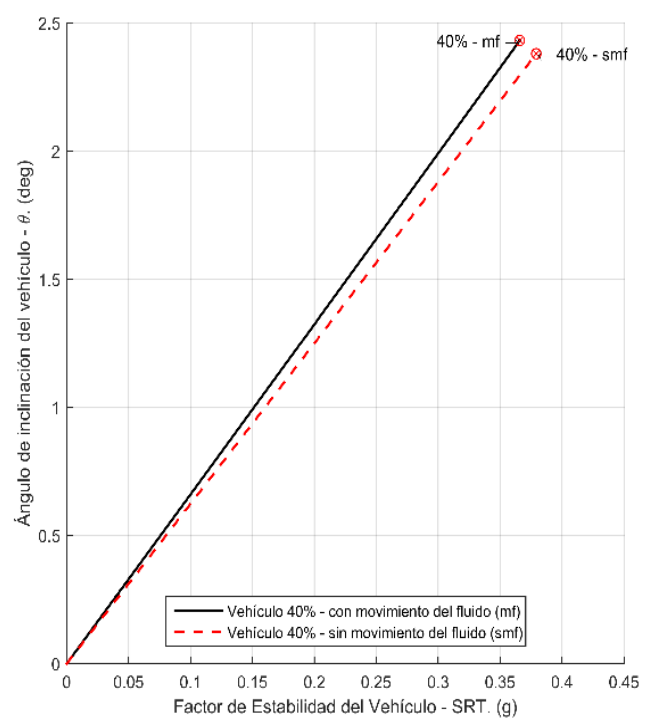

Figura 3. Factor de estabilidad $40 \%$.

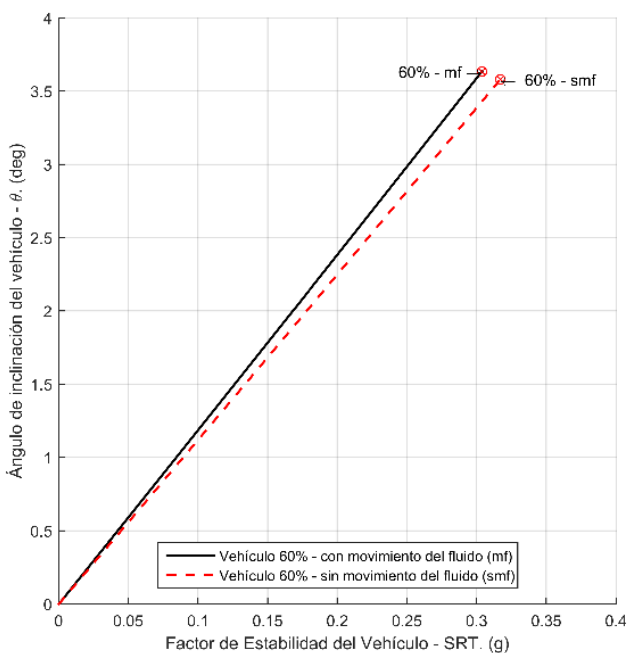

Figura 4. Factor de estabilidad 60\%.

Seguidamente, y como se puede observar en las Figuras 5 y 6 si se aumenta que la distancia de la separación lateral de la suspensión $(b)$ de $0.95 \mathrm{~m}$ a $1.062 \mathrm{~m}$, el ángulo de inclinación disminuye y la estabilidad aumenta alrededor de $1.2 \%$.

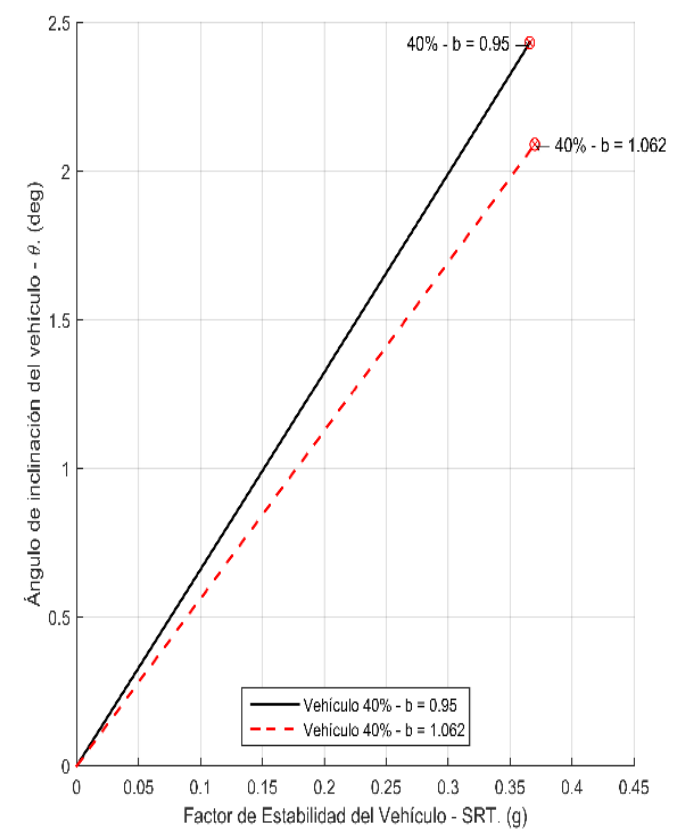

Figura 5. Factor de estabilidad 40\% - Suspensión. 


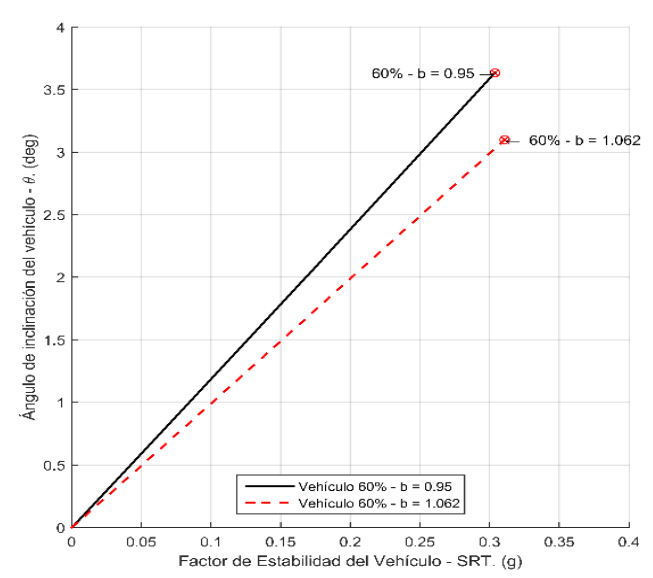

Figura 6. Factor de estabilidad 60\% - Suspensión.

\section{CONCLUSIONES}

En el presente trabajo se determinó el factor de estabilidad para vehículos tipo carro-tanque, en el cual se puede evidenciar que este factor no es solo función de la ubicación de su centro de gravedad, sino que además es función del peso fijo y móvil del vehículo.

Los resultados del estudio del trabajo del caso analizado demuestran que es importante desarrollar mecanismos que impidan el movimiento del líquido dentro del tanque - tipo rompeolas, los cuales, puedan brindar mayor estabilidad al vehículo cuando este transite por curvas o realice maniobras evasivas que impliquen riesgo de volcamiento.

En investigaciones futuras se plantea desarrollar mecanismos que puedan minimizar el movimiento del fluido transportado y por consiguiente se incremente el factor de estabilidad del vehículo.

\section{REFERENCIAS}

Çengel Yunus, A., \& Cimbala John, M. (2006). Fluid Mechanics Fundamentals and Applications. Editorial McGRAW-HILL.

ISO-14792. Heavy commercial vehicles and buses Steady state circular tests. International Organization for Starndardization. Geneva, Switzerland, 2011.

L Tangarife, M Sánchez, M Rojas (2017). Modelo de interventoría de tecnologías de información en el área de conocimiento de la gestión del alcance de PMBOK® y alineado con ISO 21500 y COBIT®. Revista Colombiana de Tecnologías de Avanzada, ISSN: 1692-7257
JEG Plaza, MAR Nuñez, (2017) Formación en competencias específicas para la industria del software colombiano. Experiencias del uso del aprendizaje basado en proyectos. Revista Colombiana de Tecnologías de Avanzada, ISSN: $1692-7257$

Moreno, G. G., Flórez, E., Peña, C. (2017a). Stability study of heavy vehicles. Revista Colombiana de Tecnologías de Avanzada ISSN: 1692-7257 - Universidad de Pamplona. v. 2, n. 30 p.p. 1 - 6, 2017.

doi: https://doi.org/10.24054/16927257.v30.n 30.2017.2756

Moreno, G. G., Nicolazzi, L., Vieira, R. S., Martins, D. (2017b). Suspension and tyres: the stability of heavy vehicles. International Journal of Heavy Vehicle Systems, ISSN online: 17415152. ISSN print: 1744-232X. v. 24 (No 4) (2017) pp. 305-326.

doi:10.1504/IJHVS.2017.087221

Prem, H., Mai, L., \& Brusza, L. (2006, June). Tilt testing of two heavy vehicles and related performance issues. In International Symposium on Heavy Vehicle Weights and Dimensions, 9th.

Ranganathan, R. (1993). Rollover threshold of partially filled tank vehicles with arbitrary tank geometry. Proceedings of the Institution of Mechanical Engineers, Part D: Journal of Automobile Engineering, 207(3), 241-244.

R García-León, E Florez, C Acevedo (2018). Caracterización térmica de mezclas de arcillas utilizadas en la fabricación de productos de mampostería para la construcción. Revista Colombiana de Tecnologías de Avanzada, ISSN: 1692-7257

Walker, H. K., Pearson, J. R. (1987). Recommended regulatory principles for interprovincial heavy vehicle weights and dimensions. Tech. rep., CCMTA/RTAC Vehicle Weights and Dimensions Study Implementation Committee Report.

Winkler, C. (2000). Rollover of Heavy Commercial Vehicles. UMTRI Rese.arch Review, The University of Michigan Transportation Research Institute, v. 31, n. 4, p. 1-20.

Woodrooffe, J. and Sweatman, P. and Arbor, A. and Middleton, D. and James, R. and Billing, J. R. (2010). National Cooperative Highway Research Program - NCHRP. Report 671. Review of Canadian Experience with the Regulation of Large Commercial Motor Vehicles. Ed. National Academy of Sciences, Washington, D.C., ISBN 978-0-309-15518-2. 